\title{
Universal Coding of the Reals using Bisection
}

\author{
Peter Lindstrom \\ Lawrence Livermore National Laboratory \\ Livermore, California \\ pl@llnl.gov
}

\begin{abstract}
We propose a simple yet expressive framework for encoding any real number as a binary string based on bisecting intervals, starting with $(-\infty,+\infty)$. Each bit of such a string represents the outcome of a binary comparison with a value contained in the interval. Our framework draws upon ideas from unbounded search and binary search, and requires only the specification of two functions: a generator for producing a monotonic sequence that brackets the number being encoded, and a refinement operator that computes an "average" of two finite numbers. Our framework is flexible enough to support many well-known representations, including Posits, ELIAs codes, logarithmic number systems, and a slightly modified version of IEEE floating point. We show that the associated generators are simple expressions given by hyperoperators. Moreover, the generality of our approach allows for exponent-less number systems based on Fibonacci and other sequences. We further analyze the probability densities associated with known and new encoding schemes and show how a compatible refinement operator and density can be derived from the bracketing sequence. This gives an almost everywhere smooth mapping between bit strings and real numbers and shows, for instance, that Posits follow a Pareto distribution. Contributions of our work include new insights into existing number systems, suggestions for how to build new, perhaps exponent-less number systems, a method for designing number systems that match a desired density, and a much simpler and verifiably correct implementation of existing representations using as few as two lines of code.
\end{abstract}

\section{CCS CONCEPTS}

- Mathematics of computing $\rightarrow$ Coding theory; Arbitraryprecision arithmetic;

\section{KEYWORDS}

Universal coding, bisection, bracketing, unbounded search, binary search, wobbling accuracy, floating point, posits

\section{ACM Reference Format:}

Peter Lindstrom. 2019. Universal Coding of the Reals using Bisection. In Conference for Next Generation Arithmetic 2019 (CoNGA'19), March 13-14, 2019, Singapore, Singapore. ACM, New York, NY, USA, 10 pages. https://doi. org $/ 10.1145 / 3316279.3316286$

Permission to make digital or hard copies of all or part of this work for personal or classroom use is granted without fee provided that copies are not made or distributed for profit or commercial advantage and that copies bear this notice and the full citation on the first page. Copyrights for components of this work owned by others than ACM must be honored. Abstracting with credit is permitted. To copy otherwise, or republish, to post on servers or to redistribute to lists, requires prior specific permission and/or a fee. Request permissions from permissions@acm.org.

CoNGA'19, March 13-14, 2019, Singapore, Singapore

(C) 2019 Association for Computing Machinery.

ACM ISBN 978-1-4503-7139-1/19/03 ..\$15.00

https://doi.org/10.1145/3316279.3316286

\section{INTRODUCTION}

An introductory computer science course will explain how to efficiently search for one of $n$ integers in a sorted list using only $\lg n$ comparisons via binary search. Indeed, if the list is contiguous, then the outcomes of binary comparisons in the search encode the integer being searched for in binary. But what if $n$ is unbounded and the list consists of arbitrary-precision real numbers?

Bentley and Yao [2] answered this question in their seminal paper on unbounded search, where several search strategies were presented. Generalized to real values $x \geq 1$, one of their strategies amounts to starting with a guess, $a=1$, and then successively doubling the guess until $x$ has been bracketed by $a \leq x<2 a$. $x$ is then located via a regular binary search on $[a, 2 a$ ) (which still may require an infinite number of comparisons). Bentley and Yao observed that their search strategies lead to binary codes of the positive integers whose bits correspond to $x<a_{i}$ (0-bit) and $x \geq a_{i}$ (1-bit), and that those codes are isomorphic to the universal codes proposed by Elias [4].

Recently [14], we extended the Elias codes from positive integers to the reals and noted the connection between the Elias $\gamma$ code and Gustafson and Yonemoto's [8] Posit floating-point like representation. Encoding and decoding Elias codes do, however, involve a non-negligible amount of code; our encoder/decoder for the exponent alone in EliAs $\omega$ is 144 lines of sparsely documented $\mathrm{C}++$ code, and the implementation is error prone. Indeed, as part of this work, we uncovered a bug in Yonemoto's own Posit implementation [17]. Moreover, each such representation requires its own specialized implementation for both encoding and decoding the fraction (which may be nonlinear [14]), exponent, regime (in the case of Posits), and sign bit, and for proper rounding and handling of under- and overflow.

In this paper, we cast coding of the reals in terms of binary comparisons in unbounded search. Our insight is that many well known number representations, as well as new ones not supported by frameworks like $[8,14]$, can be reduced to two very simple rules: one for producing a monotonic sequence, $\left\{a_{i}\right\}$, of "guesses" to bracket the number, $x$, and one for performing the binary search by recursively bisecting the bounded interval (not necessarily at its midpoint) in which $x$ has been found to lie. Equipped with these rules, no special bit manipulations are needed to encode or decode exponents, or indeed even to distinguish between exponent and fraction bits. In fact, many representations supported by our framework do not even require exponents, binary or otherwise. Nevertheless, all representations from [14], including Posits, EliAs, logarithmic number systems (LNS), floating-point representations similar to IEEE 754 [1], and others can be captured by our simple rules. As such, our framework can be thought of as a generalization and simplification of [14]. 
Our framework is based on the notion of tapered accuracy in which fewer fraction bits are allocated to very large (or very small) numbers, reserving the majority of codewords to represent real values close to one in magnitude. This is effectively achieved by using variable-length coding of exponents. Unlike IEEE floating point, which defines only a few discrete precisions, we can accommodate any precision, $p$. Each choice of $p$ results in a finite set of $2^{p}$ representable numbers that is contained in the set of $(p+1)$-bit numbers. Increasing $p$ provides not only for more precise numbers but also for higher dynamic range. Indeed, using sufficient precision, $p$, we may represent any real, and in this sense our number systems are universal. This is contrary to IEEE floating point, which by fixing the exponent length places hard bounds on the numbers that can be represented. And unlike in IEEE, no arbitrary decisions are needed to balance the number of exponent vs. fraction bits. In our framework, each number can be approximated simply by truncating it to some desired number of bits, $p$, and in practice we fix $p$ so that numbers are represented using fixed-length storage; typically $p$ is given by the underlying integer width used for the binary representation.

Our framework relies on repeated bisection of intervals and therefore encodes and decodes $p$-bit reals in $O(p)$ steps. This is not as efficient as manipulating multiple bits at a time, as done in $[13,14,17]$, and the intent of this work is not to compete with others in performance. Rather, our contribution is in the insight of this alternative view of coding reals, the new representations it affords, as well as in a significant reduction in coding effort needed to implement and experiment with new number representations. For instance, the over 100 lines of code needed just for exponent coding in Elias $\omega$ are reduced to two simple lines of code in our framework, which are sufficient to define the entire number system.

Following a description of our bisection and rounding rules, we turn our attention to how to bisect finite intervals in a manner consistent with the chosen bracketing sequence. This connects our rules with the notion of an underlying continuous probability density associated with the encoding scheme. If this density is smooth, then so is the corresponding number system. We conclude the paper with a discussion of example number systems.

\section{REPRESENTATION}

Our framework is best described in terms of recursive refinement of ordered sets, $X_{p} \subset \mathbb{R}$, of numbers generated by bisecting the intervals formed by pairs of consecutive numbers in $X_{p}$. These sets are nested: $X_{p} \subset X_{p+1}$. We will refer to $p$ as the precision, as each of the $2^{p}$ elements in $X_{p}$ is identified with a $p$-bit binary string, or equivalently a $p$-bit integer index, $y$. We refer to the process of mapping a number $x \in \mathbb{R}$ to the closest member in $X_{p}$ with index $y$ as encoding $x$; the reverse mapping from bit string $y$ to real value $x$ is referred to as decoding. Sometimes we perform arithmetic on the binary representation, $y$, which is to be understood modulo $2^{p}$.

Encoding of a number, $x$, amounts to recursive bisection of intervals, starting from $(-\infty,+\infty)$, by emitting a 0 -bit if $x$ is contained in the lower half; otherwise a 1-bit is emitted. The only degree of freedom is the choice of bisection point, which determines the binary representation of $x$ as well as the set of available numbers at finite precision $p$.

\subsection{Bootstrapping}

We begin with $X_{0}=\{ \pm \infty\}$, which by convention corresponds to the single interval $(-\infty,+\infty)=\mathbb{R}$. As in $[7,8,14], \pm \infty$ represents the "point at infinity" for projective reals. This special symbol serves multiple purposes based on context, and can be interpreted as either $-\infty$ or $+\infty$, as the result of indeterminate expressions like $0 / 0$, and as a generic $\mathrm{NaN}$ (not a number) representation.

We bootstrap our refinement process by adding these special bisection rules:

$$
\begin{aligned}
f(-\infty,+\infty) & =0, \\
f(-\infty, 0) & =-1, \\
f(0,+\infty) & =+1 .
\end{aligned}
$$

The bisection operator $f: \mathbb{R}^{2} \rightarrow \mathbb{R}$ yields a new number, $x_{m}=$ $f\left(x_{l}, x_{h}\right), x_{l}<x_{m}<x_{h}$, between $x_{l}=x\left(y_{l}\right)$ and $x_{h}=x\left(y_{h}\right)$ (not necessarily at their midpoint). This new number is assigned to the binary string $y_{m}=y_{l} 1$. As the precision is increased, we append a 0 -bit to each of $y_{l}$ and $y_{h}$. Thus, as in [8,14], $x(y)=x(y$ 0), i.e., we may append any number of 0 -bits to $y$ without changing its value.

The first rule gives us $X_{1}=\{ \pm \infty, 0\}$ while the remaining rules give us four values that are part of all representations supported by our framework: $X_{2}=\{ \pm \infty,-1,0,+1\}$ (see Fig. 1a). We add two more rules for closure under negation and reciprocation:

$$
\begin{array}{lr}
f(a, b)=-f(-b,-a) & \text { negation, } \\
f(0, b)=f\left(b^{-1},+\infty\right)^{-1} & \text { reciprocation. }
\end{array}
$$

Note that reciprocal closure generally holds only when one of the interval endpoints is in $X_{1}$. As we shall see, additional rules are needed to enforce reciprocal closure everywhere.

\subsection{Bracketing: Unbounded Search}

The above rules are sufficient only to generate 2-bit numbers. For higher precision, we must decide how to bisect arbitrary intervals. We distinguish between intervals that contain one of $\{ \pm \infty, 0\}$ as endpoint and intervals $(a, b)$, where $0<a<b<+\infty$. For the former, we apply a special bisection rule to first bracket $x \in[1, \infty)$. For $x \in(0,1)$, we apply Eq. (5) and first bracket $x^{-1}$. Similarly, if $x<0$, then we apply Eq. (4). These symmetry rules allow us to consider only the case $x \in[1, \infty)$ for the purpose of bracketing.

Given only the knowledge that $x \geq 1$, we perform an unbounded search [2] by generating a monotonic sequence $\left\{a_{i}\right\}$ of increasingly large guesses until $x \in\left[a_{i}, a_{i+1}\right)$. Starting from $a_{0}=1$, we use the following bisection rule

$$
f\left(a_{i},+\infty\right)=g\left(a_{i}\right)=a_{i+1} \quad \text { generation. }
$$

Hence, $a_{i}=g^{i}(1)$ where $g^{i}$ denotes functional power, e.g., $a_{3}=$ $(g \circ g \circ g)(1)$. For convenience, we use $a_{-i}=a_{i}^{-1}$ to denote the reciprocals of members in this sequence. (In general, $a_{-i} \neq g^{-i}(1)$ for $i>0$.) We call the function $g$ a generator for the sequence $\left\{a_{i}\right\}$. If $x \geq g\left(a_{i}\right)=a_{i+1}$, then we narrow the search interval from $\left[a_{i},+\infty\right)$ to $\left[a_{i+1},+\infty\right)$, until $x$ has eventually been bracketed in $\left[a_{i}, a_{i+1}\right)$. Fig. 1 illustrates the bracketing sequence by showing $a_{i}$ and $-a_{i}$ as red dots; their reciprocals are shown as blue dots.

We note that this generation of increasing $a_{i}$ corresponds to repeated multiplication by the useed in Posits [8] for determining the Posit regime. Moreover, each $a_{i}$ represents the largest real, $x_{\max }(p)$, 


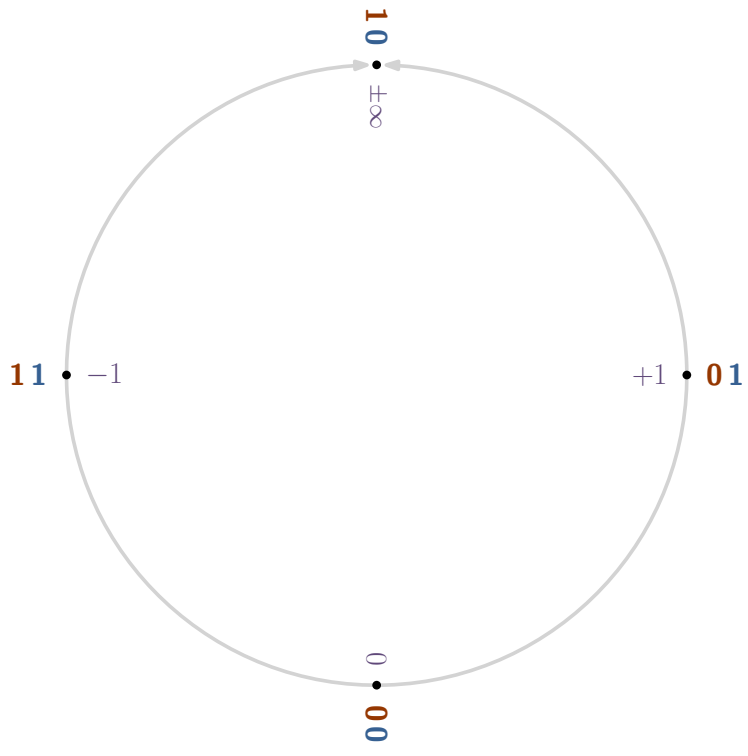

(a) $p=2$

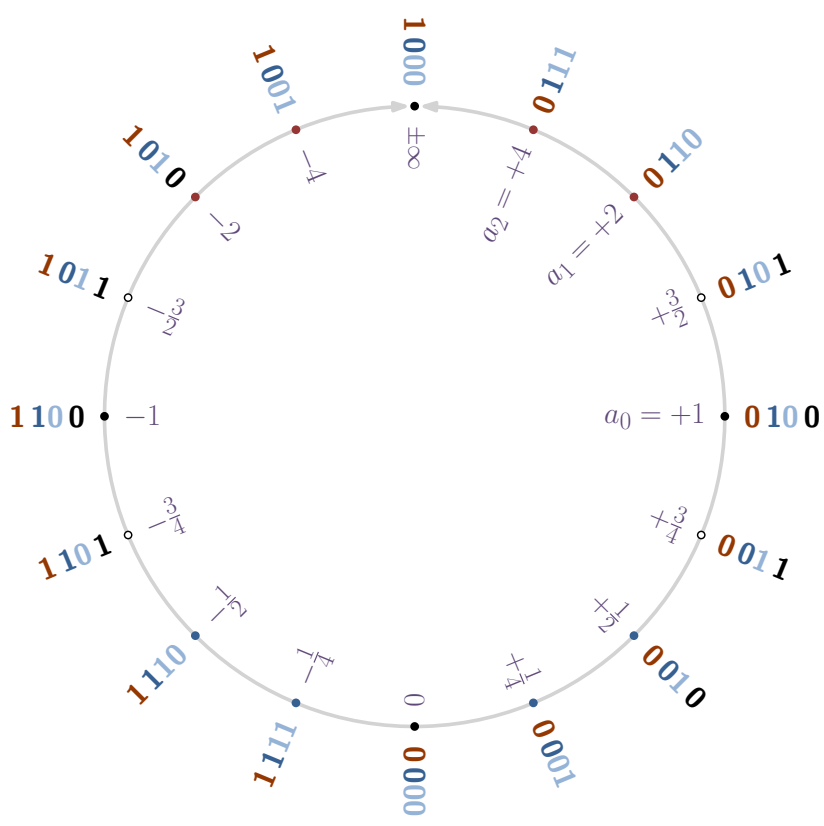

(c) $p=4$

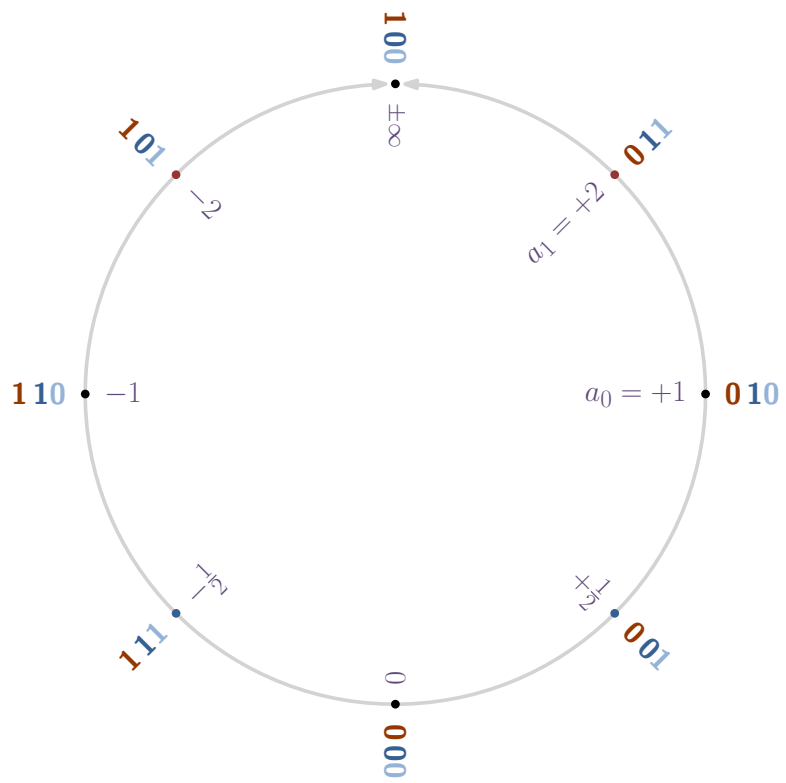

(b) $p=3$

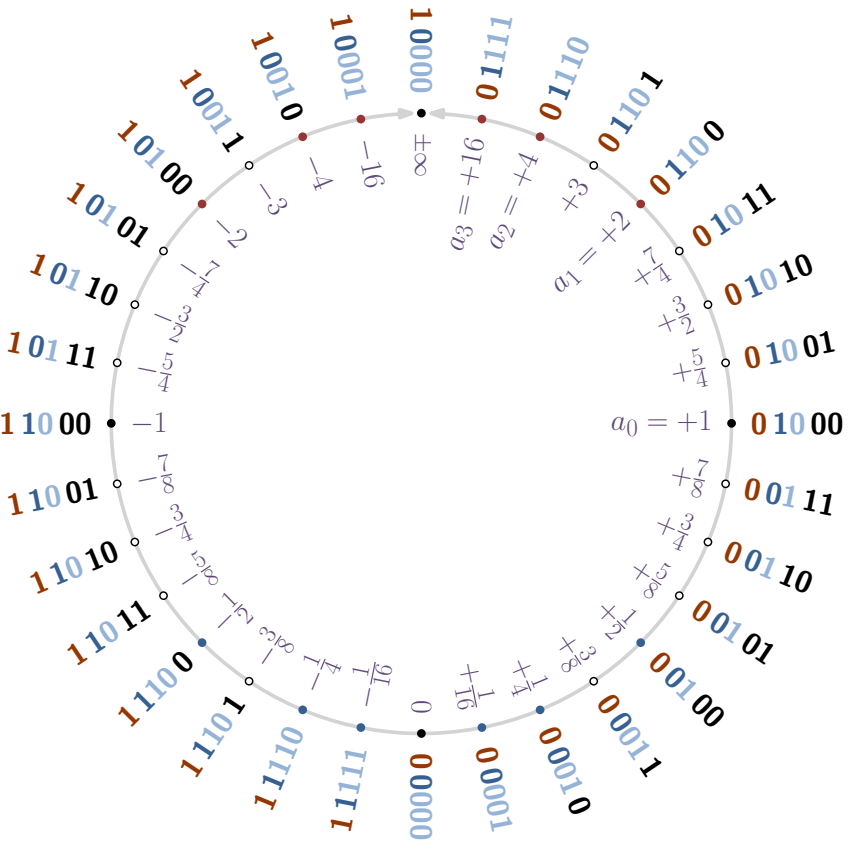

(d) $p=5$

Figure 1: Ringplot [7, 8] depicting the projective reals and how $p$-bit strings (shown on the outside) map to the reals (shown on the inside) for the URR representation [9]. Numbers ending in a zero-bit remain in place when reducing the precision by one bit. The sign bit is shown in red, exponent bits in blue, and fraction bits in black. Red dots indicate values $\left\{a_{i}\right\}$ that are part of the bracketing sequence; blue dots are their reciprocals. Hollow dots correspond to values produced via refinement. 


\subsection{Refinement: Binary Search}

At this point, $x$ has been bracketed in a bounded interval $[a, b)$. We now proceed with a binary search that recursively bisects $[a, b)$ Whereas the bracketing sequence determines the dynamic range and overall "shape" of the map between reals and their binary representation, the refinement rule governs the fine-scale shape and overall smoothness of the map.

The refinement problem reduces to choosing a midpoint $x_{m}$ for an interval $\left[x_{l}, x_{h}\right)$, with $0<x_{l}<x_{m}<x_{h}<\infty$. We may think of $x_{m}$ as being some mean of $x_{l}$ and $x_{h}$. A natural choice is $x_{m}=\left(x_{l}+x_{h}\right) / 2$, which gives rise to a linear ramp between each pair of sequence points. As we shall see later, other choices can be made to satisfy properties like reciprocal closure, varying degrees of continuity, and desired probability density.

Refinement for the number systems considered here can be expressed via the Kolmogorov mean (aka. generalized $h$-mean):

$$
K_{h}(a, b)=h^{-1}\left(\frac{h(a)+h(b)}{2}\right)
$$

for some invertible function, $h$. The usual arithmetic mean is then given by $h(x)=x$.

2.3.1 Refinement and fraction maps. Refinement is closely connected to fraction maps [14]. Fraction maps were proposed as a general mechanism for mapping fraction bits, representing numbers $r \in[0,1)$, to real values $\phi(r) \in[1,2)$ such that sign, exponent, and fraction $(s, e, r)$ bits are mapped to the real number $x=(-1)^{s} 2^{e} \phi(r)$. This mapping $\phi:[0,1) \rightarrow[1,2)$ is monotonic with $\phi(0)=1$, $\phi(1)=2$. In IEEE and Posits, $\phi(r)=1+r$ is linear and merely introduces the implicit leading one-bit. $\phi(r)=2^{r}$ ensures a smooth exponential map for systems like LNS.

In our framework, we may incorporate any fraction map by defining $f\left(x_{l}, x_{h}\right)$ appropriately. This is accomplished by noting that $f\left(x_{l}, x_{h}\right)=f\left(\phi\left(y_{l}\right), \phi\left(y_{h}\right)\right)=\phi\left(y_{m}\right)$. Thus, we simply require

$$
f\left(x_{l}, x_{h}\right)=\phi\left(\frac{\phi^{-1}\left(x_{l}\right)+\phi^{-1}\left(x_{h}\right)}{2}\right) .
$$

We immediately recognize this as an $h$-mean with $h=\phi^{-1}$.

Conversely, given the constraint $\phi^{-1}(1)=0$, if we know only the refinement rule, $f$, we may find the corresponding $\phi^{-1}$ (and $\phi$ ) by solving the functional equation (e.g., using Mathematica's RSolve)

$$
\phi^{-1}(x)=2 \phi^{-1}(f(1, x)) \text {. }
$$

For the fraction maps presented in [14], we obtain:

\begin{tabular}{ccc}
$\phi(r)$ & $f\left(x_{l}, x_{h}\right)$ & mean \\
\hline $1+r$ & $\frac{x_{l}+x_{h}}{2}$ & arithmetic \\
$2^{r}$ & $\sqrt{x_{l} x_{h}}$ & geometric \\
$\frac{2}{2-r}$ & $\frac{2}{1 / x_{l}+1 / x_{h}}$ & harmonic
\end{tabular}

We note that these are Minkowski means (aka. power means)

$$
M_{p}(a, b)=\lim _{q \rightarrow p}\left(\frac{a^{q}+b^{q}}{2}\right)^{1 / q}
$$

with $p \in\{-1,0,1\}$. Pairs of conjugate fraction maps [14] that ensure reciprocal closure may be constructed using $p$-means of opposite sign, e.g., the arithmetic $(p=1)$ and harmonic $(p=-1)$ mean give rise to the conjugate linear and reciprocal maps. As in [14], we allow for different refinement rules on $(0,1)$ and $(1, \infty)$ to satisfy reciprocal closure.
2.3.2 Hyper mean. Posits with at least one exponent bit and Elias $\delta$ and $\omega$ generate sequences that grow faster than by a factor of two. Because they all encode binary exponents and use linear fraction maps, resulting in arithmetic mean refinement within a binade, their corresponding refinement rules are different when the interval spans more than one binade, i.e., when $x_{h}>2 x_{l}$. When this happens, $x_{l}$ and $x_{h}$ are both powers of two, and for Posits and ELIAS $\delta$ one can show that one should then average their exponents, corresponding to taking the geometric mean $x_{m}=\sqrt{x_{l} x_{h}}$ [8]. For ELIAS $\omega$, we may even have $\log _{2} x_{h}>2 \log _{2} x_{l}=\log _{2} x_{l}^{2}$. We may then repeat the process by taking the geometric mean of exponents.

To handle this in a generic way, we propose the hyper mean given by

$$
H(a, b)= \begin{cases}-H(-a,-b) & \text { if } a<0 \wedge b<0, \\ \frac{a+b}{2} & \text { if } a=0 \vee b=0 \vee \frac{1}{2} \leq \frac{a}{b} \leq 2, \\ 2^{H\left(\log _{2} a, \log _{2} b\right)} & \text { otherwise. }\end{cases}
$$

\subsection{Rounding}

Because the set of representable values, $X$, is finite, we need a way of rounding continuous reals from an infinite set to a nearby value from this discrete set. We use $\mathrm{fl}(x)$ to denote the discrete value that $x$ rounds to. We distinguish two cases: when the two closest discrete values are both finite and nonzero and when one of them is not.

2.4.1 Rounding finite values. As in [14], rounding $x$ begins by bracketing it between the two closest discrete values $x_{l} \leq x<x_{h}$. We then compute $x_{m}=f\left(x_{l}, x_{h}\right) . x$ is rounded to $x_{l}$ if $x<x_{m}$ and to $x_{h}$ if $x>x_{m}$. In case $x=x_{m}$, there is a tie. As is common, we use "banker's rounding," also known as "round to nearest, ties to even," to break the tie. That is, we round to the number whose binary representation, $y_{l}$ or $y_{h}$, at the current precision ends in a zero-bit.

2.4.2 Over- and underflow. Rounding of values that exceed $x_{\max }$ or are smaller than $x_{\min }$ require special attention, as such values fall outside the range of representable finite non-zero values. We allow the user to select one of three modes: round to $x_{\max }\left(x_{\min }\right)$; round to $\pm \infty(0)$; or use the same rounding rule as used for finite non-zero values, i.e., by comparing the value being rounded to the next sequence point $a_{p-1}\left(a_{1-p}\right)$.

2.4.3 Gradual underflow. The IEEE standards committee [1] recognized that if all fractions have an implied leading one-bit, then as one traverses the representable numbers toward zero, one reaches a steep cliff just before zero, where the few closest numbers to zero are $\left(x_{0}, x_{1}, x_{2}, x_{3}\right)=\left(0,2^{e}, 2^{e}\left(1+2^{-n}\right), 2^{e}\left(1+2 \times 2^{-n}\right)\right)$. Here $n$ is the number of stored significand bits and $e$ is the smallest representable exponent. In other words, the drop from $x_{1}$ to $x_{0}$ is $2^{n}$ ( $n=52$ for double precision) times larger than the gradual slope from $x_{2}$ to $x_{1}$. Such a sharp discontinuity in the representation is clearly unwanted.

Gradual underflow circumvents this problem through the introduction of subnormals in IEEE. For the smallest representable exponent, $e$, the implicit leading fraction bit is zero for the subnormals, which results in a gradual ramp extending all the way to zero. 

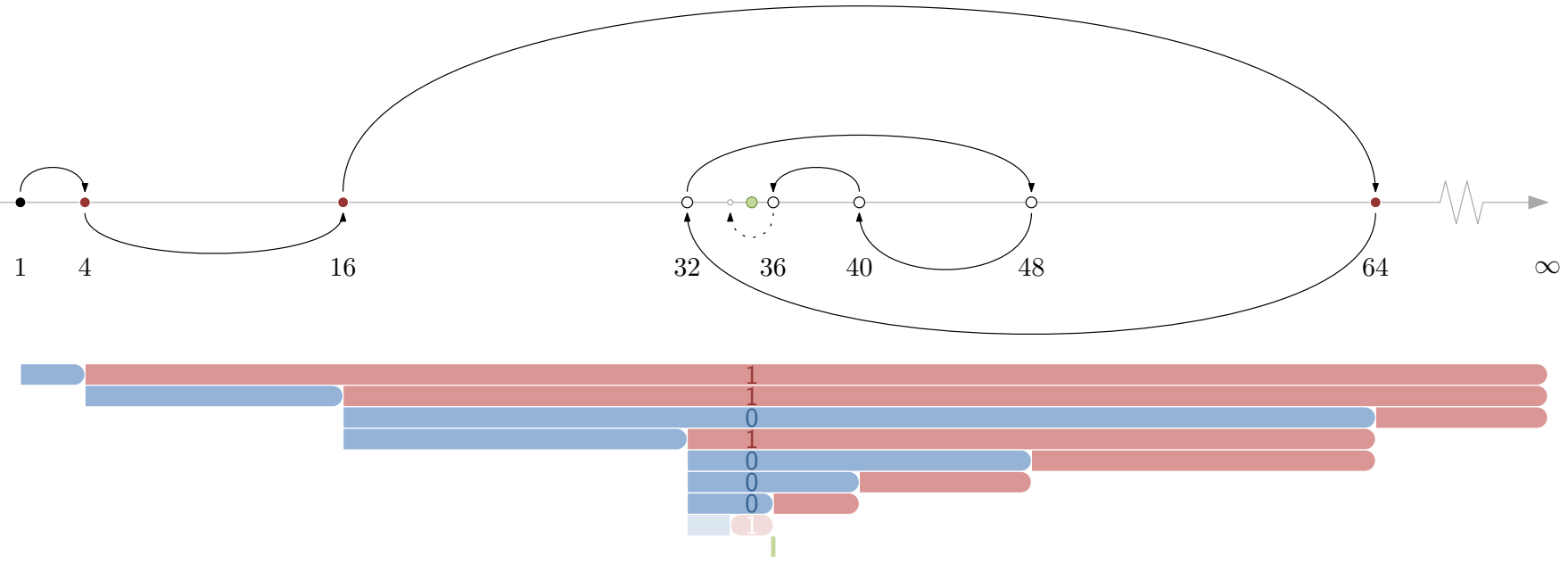

Figure 2: To encode a value, $x \geq 1$, we make a series of binary comparisons of $x$ with a monotonic number sequence $a_{i}$. Once $x \in\left[a_{i}, a_{i+1}\right)$ has been bracketed, this interval is recursively narrowed using (not necessarily uniform) bisection. In this example, $x=35$ is encoded by PosIT(1), with a bracketing sequence of $\left(4^{0}, 4^{1}, 4^{2}, 4^{3}, \ldots\right)$. $x$ is shown in green; sequence points, $a_{i}$, are shown in red; refinement points are hollow; the rounding point is small and hollow. The arrows indicate the order of comparisons. The bisected intervals are shown below, with the emitted bits indicating if the lower $(0=$ blue $)$ or upper $(1=$ red) half with respect to the bisection point was selected. The end result is $y(35)=0111010001 \ldots$ that, for 9-bit precision, is rounded up to $y(\mathrm{fl}(x)=36)=011101001$. The prefix 01 indicates that $x \in[1, \infty)$.

The problem of abrupt overflow in IEEE is largely an artifact of fixing the exponent length, which results in hard lower and upper bounds on representable values. Because of universality, such bounds are absent in our framework and in related representations that utilize tapered accuracy $[8,14]$. Nevertheless, abrupt underflow is still possible. We here present a condition needed to satisfy gradual underflow in our framework.

Let $x_{0}=0, x_{1}=x_{\min }$, and $x_{2}$ be the smallest number succeeding $x_{\min }$. We say that gradual underflow is satisfied if $x_{1}-x_{0} \leq x_{2}-x_{1}$. Since $x_{0}=0$, this reduces to $2 x_{1} \leq x_{2}$, and because $x_{1}$ and $x_{2}$ are both reciprocals from the bracketing sequence, this in turn implies that gradual underflow is achieved if $2 a_{i} \leq a_{i+1}$. In other words, the bracketing sequence must increase at least exponentially with base two or higher. All Posits and Elias codes satisfy this constraint, although we will later present number systems that do not.

\subsection{Encoding and Decoding}

We are finally ready to describe how bit strings, $y$, are decoded to their corresponding reals, $x(y)$, and how reals, $x$, are encoded. Decoding and encoding are essentially done in the same way using recursive bisection. The idea is to narrow an interval, $\left[x_{l}, x_{h}\right)$, that initially equals $[-\infty,+\infty)$, so that the number, $x$, remains bracketed: $x_{l} \leq x<x_{h}$. Bisection around $x_{m}=f\left(x_{l}, x_{h}\right)$ leads to two smaller intervals: $\left[x_{l}, x_{m}\right),\left[x_{m}, x_{h}\right)$. The lower half corresponds to a zerobit; the upper half to a one-bit. This results in a sequence of bits that encode which half is chosen at each step.

When decoding, we process one bit at a time, from left to right, and narrow the interval accordingly. When encountering a zero-bit, we replace $x_{h}$ with the bisection point $x_{m}$; otherwise, we replace $x_{l}$ with $x_{m}$. The final value is given by the lower end, $x_{l}$, of the interval.
When encoding, we compare $x$ to the bisection point, $x_{m}$. If $x<x_{m}$, then we replace $x_{h}$ with $x_{m}$ and emit a zero-bit; otherwise we replace $x_{l}$ with $x_{m}$ and emit a one-bit. When we exhaust the available precision, we are left with an interval $\left[x_{l}, x_{h}\right)$, and we must round $x$ to one of these endpoints. As discussed in Section 2.4, we then generate another bisection point, $x_{m}$, and round based on the outcome of a comparison with $x$.

Finally, our binary representation, $y$, is treated as a two's complement signed integer so that comparison between two reals can be done directly on the two's complement representation. This, however, requires that $x=0$ maps to $y=000 \ldots 0$, but without modification $x$ would map to $y=100 \ldots 0$. Hence, we flip the leftmost bit of $y$ after encoding and before decoding.

As a consequence of our rules, when $1 \leq a_{i} \leq x<a_{i+1}$, the prefix of $x$ 's binary representation, $y$, equals $011^{i} 0$. In other words, we encode in unary ( $i$ ones terminated by a zero) the number of applications, $i$, of $g$ to reach $a_{i}$. The bits in this prefix of $y$ correspond to the outcomes of comparisons of $x$ with the sequence $(0,1=$ $\left.a_{0}, \ldots, a_{i}, a_{i+1}\right)$ and assert that $0<1=a_{0} \leq \cdots \leq a_{i} \leq x<a_{i+1}$. Similarly, when $0<a_{i-1} \leq x<a_{i} \leq \cdots \leq a_{0}=1$ with $i \leq 0$, the prefix of $y$ equals $000^{-i} 1$. Thus, commonly occurring numbers near one require not only fewer bits to bracket, leaving more bits for refinement, but the intervals $\left[a_{i}, a_{i+1}\right)$ also tend to be narrower near one, which results in further increased accuracy. This property is often referred to as tapered accuracy [15].

\section{NUMBER DISTRIBUTIONS}

As is well-known for universal codes for (positive) integers [4], any coding scheme has an associated discrete probability distribution associated with it for which the scheme is optimal (in the Shannon entropy sense). This distribution should ideally reflect the likelihood 
of numbers occurring in the intended application. Such an argument can be extended to our universal codes for the reals, for which the underlying probability density, $f(x)$, associated with a random variable $X$ is continuous. That is, when bisecting an interval $[a, b)$, we do so at a point $c$ such that $F(c)-F(a)=F(b)-F(c)$, where $F$ denotes the cumulative distribution function. In other words, we bisect such that $P(a \leq X<c)=P(c \leq X<b)$, which ensures that we assign the same number of codes to equally probable intervals; codes representing $[a, c)$ begin with 0 , while codes representing $[c, b)$ begin with 1 .

We here establish a connection between density and coding scheme in the sense that the density implies both the bracketing sequence and refinement scheme. Our approach is to find a continuous, smooth cumulative distribution $F(x)$ that agrees with the discrete bracketing sequence associated with a coding scheme. Given $F$, we then solve $F(c)-F(a)=F(b)-F(c)$ for $c$, giving

$$
c=F^{-1}\left(\frac{F(a)+F(b)}{2}\right),
$$

which again is a Kolmogorov mean.

We use $F_{00}(x)=F(x \mid 0 \leq X<1)=4 F(x)-2$ and $F_{01}(x)=$ $F(x \mid 1 \leq X)=4 F(x)-3$ to denote truncated CDFs whose subscripts correspond to the binary prefixes associated with values in these ranges. Because the numbers in the bracketing sequence are closed under reciprocation, we have

$$
F_{00}(x)=1-F_{01}\left(x^{-1}\right),
$$

which then implies that the resulting representation is completely closed under reciprocation.

Due to our unary representation of the bracket index, $i$, all of our coding schemes satisfy

$$
\begin{array}{ll}
F_{00}\left(a_{i}\right)=2^{i} & \forall i \leq 0, \\
F_{01}\left(a_{i}\right)=1-2^{-i} & \forall i \geq 0 .
\end{array}
$$

Hence, given a closed-form expression for $a_{i}$ (e.g., from Table 1), we may solve for $i$ in terms of $a_{i}$. We then relax the requirement that $i$ be an integer and replace $a_{i}$ with any $x \in[1, \infty)$. For instance, when $a_{i}=b^{i}$, we have $i=\log _{b} a_{i}=\log _{b} x$. Plugging this into Eq. (15) leads to $F_{01}(x)=1-2^{-\log _{b} x}=1-x^{-1 / \log _{2} b}$ for all $x \in[1, \infty)$. Using Eq. (12), the associated refinement scheme can then be shown to be a power mean, $M_{p}$, with $p=-1 / \log _{2} b$.

Note that this solution to refinement differs from simply plugging in a fractional $i$ in the expression for $a_{i}$. For example, using $a_{i}=2^{i}$, a naïve solution gives $a_{1 / 2}=2^{1 / 2}=\sqrt{2}$ while our derivation gives $a_{1 / 2}=M_{-1}\left(2^{0}, 2^{1}\right)=\frac{4}{3}$.

\subsection{Relative Accuracy}

The density of numbers

$$
f=\frac{d F}{d x} \approx \frac{\Delta F}{\Delta x}=2^{-p} \frac{\Delta y}{\Delta x}
$$

dictates how accurately we may represent $x$. Here $\Delta y$ is the number of distinct representable $p$-bit values in a given interval of width $\Delta x$. We measure the relative accuracy in number of bits as

$$
\alpha(x)=\log _{2}(|x| f(x)) .
$$

Letting $\Delta x$ be the width of the interval of values that round to $\mathrm{fl}(x)$, $\Delta y=1$ and we obtain the finite-precision approximation

$$
\alpha(x) \approx \log _{2}\left(\frac{\Delta y}{2^{p}} \frac{|x|}{\Delta x}\right)=\log _{2}\left(\frac{|x|}{\Delta x}\right)-p .
$$

The reciprocal $\frac{\Delta x}{x}$ is a measure of relative error in $x$ due to rounding. In the limit, as the precision $p$ approaches infinity, $2^{-p} \Delta y \rightarrow d F$, and we obtain Eq. (17), a precision-independent measure of relative accuracy in terms of the continuous density $f . \alpha$ is in general negative and can be thought of as the overhead in number of bits lost to coding the sign and exponent (or sequence index, $i$ ).

To provide more intuition, we now discuss distributions and accuracies of example representations (see also Table 1). We will see how the natural refinement rule (Eq. (12)) eliminates what is known as wobbling accuracy $[7,8,10]$. For simplicity, we assume $x \geq 1$ and present only $F_{01}$ and $f_{01} . F$ and $f$ are obtained using Eq. (13) and $f(x)=f\left(x^{-1}\right)=f(-x)$.

\subsection{Extended UnARY}

We begin by considering a generalization of unary code that represents non-negative integers, $x$, as $y(x)=01^{x} 0$ (with the leading zero being the sign bit). The bracketing sequence for UNARY is $(1,2,3, \ldots)$. Plugging $x=a_{i}=i+1$ into Eq. (15) and differentiating, we obtain

$$
\begin{aligned}
F_{01}(x) & =1-2^{1-x}, \\
f_{01}(x) & =\ln (2) 2^{1-x}, \\
\alpha(x) & =\eta-1+|\lg | x||-2^{|\lg | x||} .
\end{aligned}
$$

where $\eta=\lg (\ln (2))$. This corresponds to the Laplace distribution (with $\lambda=1 / \ln 2$ ) when $|x| \geq 1$, but not when $|x|<0$ due to our insistence on reciprocal closure. The quantile function is given by $F_{01}^{-1}(y)=1-\log _{2}(1-y)$. Solving Eq. (12), we find as refinement operator $c=1-\log _{2}\left(2^{-a}+2^{-b}\right)$ when $1 \leq a<b$.

\subsection{Posits and Elias $\gamma$}

In the case of $\operatorname{Posit}(m)$, with $\operatorname{Posit}(0)$ identical to Elias $\gamma$ [14], we may easily verify that

$$
\begin{aligned}
F_{01}(x) & =1-x^{-2^{-m}}, \\
f_{01}(x) & =2^{-m} x^{-2^{-m}-1}, \\
\alpha(x) & =-2-m-2^{-m}|\lg | x|| .
\end{aligned}
$$

This is a type- 1 Pareto distribution with minimal value 1 and shape parameter $2^{-m}$. Using the same approach as for UNARY, we find that the natural refinement scheme on $[1, \infty)$ is given by the power mean (Eq. (10)) with $p=-2^{-m}$. That is, $p \in\left\{-1,-\frac{1}{2},-\frac{1}{4},-\frac{1}{8}, \ldots\right\}$. On $(0,1)$, the conjugate refinement scheme simply reverses the sign of $p$, so that $p=2^{-m}$.

Using these conjugate schemes instead of the usual piecewise linear interpolation employed by Posits, we obtain "NATURALPosits." Whereas the refinement employed by Posits implies a piecewise constant density, NaturalPosits have a smooth density without wobbling accuracy (Fig. 4). For instance, when $m=0, f_{01}(x)=x^{-2}$. Interestingly, NATURALPosits $(0)$ were discovered by us independently in earlier work (see [14, Eq (6)]), but in an ad hoc manner without the insight and derivation provided here. 


\begin{tabular}{lcccccc} 
representation & $a_{1}$ & $a_{6}$ & $a_{i}$ & $g(x)$ & $f(a, b)$ & $f^{*}(a, b)$ \\
\hline UnARY & 2 & 7 & $i+1$ & $x+1$ & & $K_{2^{-x}}$ \\
\hline base $\phi$ & $\phi$ & $\phi^{6} \approx 17.9$ & $\phi^{i}$ & $\phi x$ & & $M_{-1 / \log _{2} \phi}$ \\
FIBONACCI & 2 & 21 & $F_{i+2}$ & $\operatorname{round}(\phi x)$ & $M_{1}$ & $?$ \\
ELIAS $\gamma$ & 2 & 64 & $2^{i}$ & $2 x$ & $M_{1}$ & $M_{-1}$ \\
base $2^{m}$ & $b=2^{m}$ & $b^{6}$ & $b^{i}$ & $b x$ & $M_{0}$ & $M_{-1 / m}$ \\
base $2^{m}+1$ & $b=2^{m}+1$ & $b^{6}$ & $b^{i}$ & $b x$ & $M_{1}$ & $M_{-1 / \log _{2}\left(2^{m}+1\right)}$ \\
Posit $(m)$ & $b=2^{2^{m}}$ & $2^{6 \times 2^{m}}$ & $b^{i}$ & $b x$ & $H$ & $M_{-2^{-m}}$ \\
\hline URR & 2 & $2^{32}$ & $2^{\left.2^{i-1}\right\rfloor}$ & $\max \left\{2, x^{2}\right\}$ & $H$ & $K_{1 / \log _{2}(x)}$ \\
ELIAS $\delta$ & 2 & $2^{63}$ & $2^{2^{i}-1}$ & $2 x^{2}$ & $H$ & $K_{\log _{2}(x) / \log _{2}(2 x)}$ \\
\hline ELIAS $\omega$ & 2 & $2^{2^{65536}}$ & $2 \uparrow \uparrow i$ & $2^{x}$ & $H$ & $?$ \\
\hline $\operatorname{FP}(m)$ & $2^{2^{m-2}}$ & see text & see text & $H\left(x, 2^{2^{m-1}}\right)$ & $H$ & $M_{0}$ \\
$\operatorname{LNS}(m)$ & $2^{2^{m-2}}$ & $2^{63 \times 2^{m-7}}$ & $2^{2^{m-1}\left(1-2^{-i}\right)}$ & $2^{2^{m-2}} \sqrt{x}$ & $M_{0}$ & $M_{0}$ \\
\hline
\end{tabular}

Table 1: The generator, $g$, produces the sequence $a_{i+1}=g\left(a_{i}\right)$, with $a_{0}=1 . a_{6}$ is the largest value representable in an 8-bit “minifloat” (aka. quarter precision). $f^{*}$ represents natural refinement and is presented for $x \geq 1$.

\subsection{URR, Elias $\delta$, and Elias $\omega$}

In [14], we presented a slight variation on Elias' original $\delta$ code [4] that differs in how the $\gamma$ code, used by $\delta$ to encode the exponent of $x$, is extended from positive to non-negative integers. That version of $\delta$, which is identical to Hamada's URR representation [9], corresponds to a generator $g(x)=x^{2}$ with a special bootstrapping rule $g(1)=2$. The original Elias $\delta$ code can be shown to use $g(x)=2 x^{2}$. Its lack of special rule simplifies the derivation of its density, and we use Elias' original definition of $\delta$ here.

For Elias $\delta$, it is easy to show that

$$
\begin{aligned}
F_{01}(x) & =\frac{\lg (x)}{1+\lg (x)}, \\
f_{01}(x) & =\frac{\ln (2)}{x \ln (2 x)^{2}}, \\
\alpha(x) & =-\eta-2(1+\lg (1+|\lg | x||)),
\end{aligned}
$$

with fraction map $\phi(r)=2^{r /(2-r)}$ when $x \geq 1$. This distribution is similar to the log-Cauchy distribution. The natural refinement rule is a Kolmogorov mean given by Eqs. (12) and (25)

As for Elias $\omega, a_{i}=2 \uparrow \uparrow i$ is given by tetration, which lacks a commonly accepted consistent definition for non-integral $i$. Therefore, we generally know $F$ only at discrete points, and the derivation of a natural refinement scheme based on "super logarithms" is an open issue. Using linear refinement, $M_{1}$, we can of course complete $F$ and derive a piecewise constant density.

\subsection{IEEE and LNS}

IEEE 754 floating point [1] is the number representation implemented by virtually all contemporary processors. With its unbalanced exponent range, sign-magnitude representation, and exceptions for representing NaNs and subnormals, IEEE does not fit directly into our framework. Later, we will present a simplified representation that serves as a good proxy for IEEE.

IEEE(11)-double precision floating point with 11 bits of exponenthas a bracketing sequence that begins with $a_{0}=2, a_{1}=2^{513}$. To appreciate how inconceivably large this next "guess" after 2 is, consider that the universe holds only about $2^{270}$ atoms and spans roughly $2^{205}$ Planck lengths (the smallest meaningful unit of length). According to IEEE, the best guess after 2 is a number nearly one googol $\left(10^{100}\right)$ universes in size!

Common numbers like $x=5$ are grossly overestimated by IEEE. After being bracketed in $\left[2,2^{513}\right)$, IEEE(11) has to backtrack in its binary search, resulting in a lengthy sequence of guesses $\left(2,2^{513}, 2^{257}, 2^{129}, 2^{65}, 2^{33}, 2^{17}, 512,32,8,4\right)$ when searching for $x$, which wastes precious bits. By comparison, our tapered representations begin with $a_{0}=1$ and then build up an accelerating sequence, such as $(1,2,4,8)$ for Elias $\gamma$, and therefore quickly find a tight bound for frequently occurring numbers around 1.

In terms of number distribution, $\operatorname{IEEE}(11)$ allocates half of all bit strings to represent numbers larger than $2^{513}$ or smaller than $2^{-511}$ in magnitude-numbers that rarely occur in most applications. And unlike the other representations presented here, whose dynamic range increases with precision, $\operatorname{IEEE}(11)$ imposes an unscalable wall at $2^{1024}$, where the numbers inexplicably come to an abrupt end-regardless of how many bits of precision are available.

In [14] we presented a slight variation on IEEE that uses (1) no special exponents for subnormals or NaNs, (2) two's complement instead of sign-magnitude, which avoids two distinct representations for zero, and (3) an exponent bias such that $a_{0}=1$. Referred to as BINARY in [14] for its use of a fixed-length binary encoding of the exponent, we will here denote this IEEE-like representation by $\operatorname{FP}(m)$, where $m$ is the number of exponent bits. $\mathrm{FP}(m)$ has an associated bracketing sequence that is generated by $g(x)=H\left(x, 2^{2^{m-1}}\right)$, which for $m=11$ gives a sequence $\left(1,2^{512}, 2^{768}, 2^{896}, \ldots\right)$ that is bounded by $2^{2^{m-1}}=2^{1024}$. In numerical experiments, we found FP to perform identically to IEEE.

Like IEEE, FP uses linear interpolation for refinement, which results in wobbling accuracy that is avoided using natural refinement. As it turns out, the natural refinement scheme for FP is given by $M_{0}$, i.e., the geometric mean. This gives rise to what is known as the logarithmic number system [11]. LNs $(m)$, uses a fixed-length $m$-bit signed exponent and exponential fraction map. We may write the LNs bracketing sequence as

$$
a_{i}=2^{2^{m-1}\left(1-2^{-i}\right)} .
$$




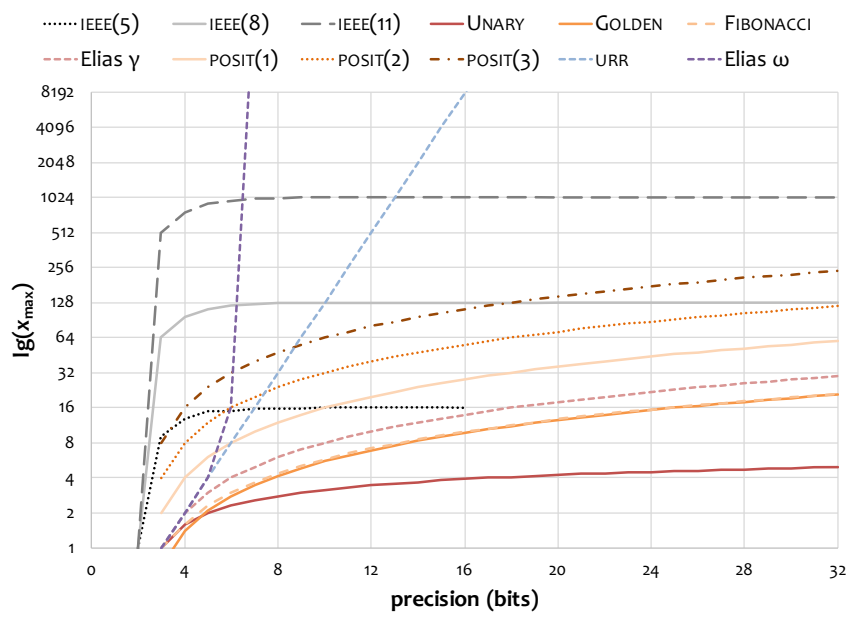

Figure 3: Dynamic range as a function of precision. $x_{\max }(p)$ is the largest value that can be represented at precision $p$. Unlike IEEE, our representations have no set ceiling.

We thus have on $1 \leq x<2^{2^{m-1}}$

$$
\begin{aligned}
F_{01}(x) & =2^{1-m} \log _{2} x, \\
f_{01}(x) & =\frac{2^{1-m}}{\ln (2) x}, \\
\alpha(x) & =-\eta-1-m .
\end{aligned}
$$

This is know in statistics as the reciprocal distribution.

\subsection{Discussion}

One advantage of our density-based approach is that it gives us a representation with an associated smooth density defined everywhere, which in turn gives us an everywhere (except, perhaps, at $\{-1,0,1\})$ smooth mapping from bit strings to numbers without any unwanted kinks or wobbling precision. This is unlike the sawtooth like accuracy inherent in piecewise linear representations like IEEE and Posits (Fig. 4). The closed-form distribution given by natural refinement may be used to analyze properties of the number scheme, such as its accuracy, or of related representations like Posits that share the same bracket sequence. Our construction also answers the question of how to choose a refinement scheme that is "compatible" with the chosen bracket sequence. Although the resulting representations can be computationally expensive to translate to and from, they may find utility in low-precision computations, where lookup tables are practical, or when accuracy takes precedence over computational efficiency.

We finally note that on $x \in(-\infty,+\infty), 0 \leq F(x) \leq 1$ in effect is the encoding function that maps $x$ to $\hat{y}=\frac{y}{2^{p}}$. Viewed differently, our $2^{p}-1$ finite values are given by $F^{-1}\left(2^{-p} y\right)$ with $y \in\left\{1, \ldots, 2^{p}-1\right\}$ Thus, any monotonically increasing function, $F: \mathbb{R} \rightarrow[0,1]$, implies an associated number representation for $\mathbb{R}$. To fit within our framework, such a function should satisfy $F(x)+F(-x)=1$ (for closure under negation) and $2\left|F(x)+F\left(x^{-1}\right)-1\right|=1$ (for closure under reciprocation). Example sigmoids (used often in neural networks) that meet this requirement are softsign [6] and $\tan ^{-1}$.

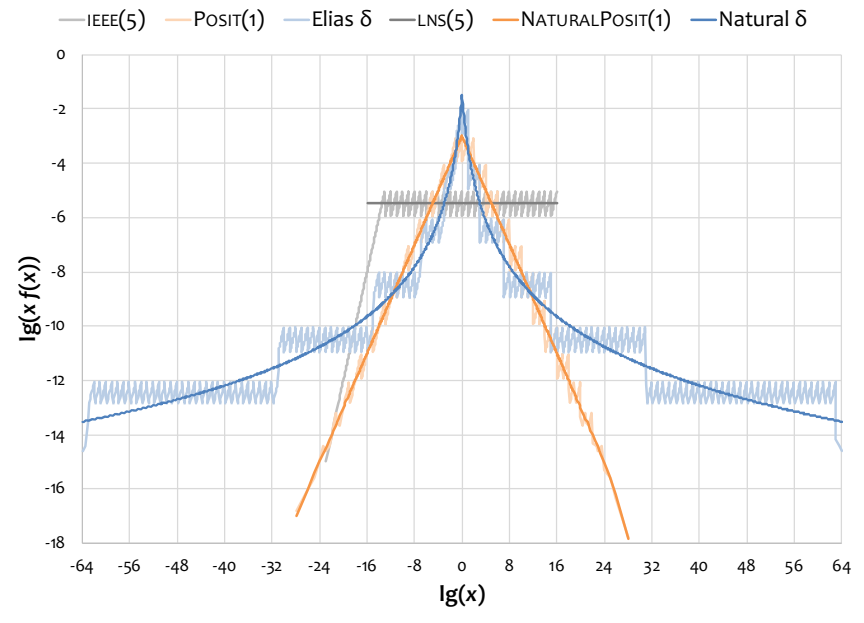

Figure 4: Relative accuracy (in bits) for 16-bit IE Ee, Posit(1), and Elias $\delta$. Posits and Elias $\delta$ demonstrate tapered accuracy that is maximal at $x=1$. The piecewise linear representations illustrate significant wobbling accuracy, which is eliminated using natural refinement, as evidenced by the corresponding natural representations.

\section{EXAMPLES}

We here discuss known number systems that can be expressed in our framework, as well as others that to our knowledge are new (refer to Table 1 and Fig. 3). Several of these do not fit into the family of representations proposed in [14], which requires explicit separation into sign, binary exponent, and fraction.

The insight that number systems can be designed via the specification of sequences produced by an associated generator leads one to immediately consider simple generators and sequences, such as Fibonacci. Perhaps the simplest generator is $g(x)=x+1$. We call the corresponding number representation extended UNARY since it encodes positive integers in unary code, $y(x)=01^{x} 0$ (the leading zero is the sign bit), while extending to all of $\mathbb{R}$. Table 1 lists the associated natural interpolant for UnARY. We note that the slowly increasing sequence $a_{i}=i+1$ results in abrupt underflow, which otherwise might make UNARY suitable for low dynamic range applications, e.g., neural networks.

UNARY together with URR and ELIAS $\gamma$ and $\omega$ have generators with successively increasing growth rate. We recognize their associated expressions for $a_{i}$ as the hyperoperations

\begin{tabular}{lccll} 
system & $g(x)$ & $a_{i}$ & operator & note \\
\hline UNARY & $x+1$ & $i+1$ & hyper-0 & succession \\
ELIAS $\gamma$ & $2 x$ & $2^{i}$ & hyper-3 & exponentiation \\
URR & $x^{2}$ & $2^{2^{i-1}}$ & lower hyper-4 & \\
ELIAS $\omega$ & $2^{x}$ & $2 \uparrow \uparrow i$ & upper hyper-4 & tetration
\end{tabular}

using Knuth notation [12] for tetration. The omission of hyper-1 (aka. addition) and hyper-2 (aka. multiplication) suggests a gap in representations. However, these operators yield sequences $a_{i}=2+i$ and $a_{i}=2 i$ that have the same growth rate as UNARY. 
From Table 1, we notice several new representations with exponential bracketing sequences, but which do not use binary exponents and therefore cannot be expressed in [14]. One such representation is FIBONACCI, which uses the well-known sequence $a_{i+1}=a_{i}+a_{i-1}=\operatorname{round}\left(\phi a_{i}\right)$, where $\phi=(\sqrt{5}+1) / 2$ is the golden ratio. This representation should not be mistaken for Fibonacci codes [16], which express integers as sums of Fibonacci numbers. In the limit, the ratio of consecutive Fibonacci numbers approaches $\phi$, which we may also use as exponential base for our sequences (i.e., $a_{i+1}=\phi a_{i}$, with no rounding). This allows for a smooth representation whereas FiBONACCI has the advantage of representing only integers and rationals, if so desired. Note that base $\phi$ is unrelated to "phinary" [3], which expresses numbers as sums of powers of $\phi$. The various identities surrounding $\phi$ makes this an interesting base to work in, and more work is needed to determine a suitable refinement scheme for it and for FibONAcCi.

Whereas $\operatorname{Posit}(m)$ allows for varying bases $b=2^{2^{m}}$, the set of reasonable choices for $m$ are few (usually $m \leq 3$ ). Our framework easily handles any base. One example is $b=2^{m}+1 \in\{2,3,5,9, \ldots\}$ coupled with a linear fraction map, $\phi(r)=1+r$, where $r \in\left[0,2^{m}\right)$. Hence, $x=\left(2^{m}+1\right)^{i}(1+r)$, where $r$ denotes the fraction bits generated by refinement. Another choice is $b=2^{m}$ with $M_{0}$, which implies $x=2^{m i} 2^{m r}=2^{m(i+r)}$ with $r \in[0,1)$.

\section{IMPLEMENTATION}

Appendix A lists Mathematica code for our framework. We provide functions to encode a real number as a binary string. Such strings can be converted to integers via FromDigits[string, 2]. Decoding can be done either from a bit string or a $p$-bit integer $\left(\bmod 2^{p}\right)$. Bisection of intervals is performed by the bisect function. Two user-supplied functions are needed that uniquely define the number system: $g(x)$ and $f(a, b)$.

We have also developed a $\mathrm{C}++$ implementation (not included here) that handles encoding, decoding, bisection, and rounding. As with the Mathematica implementation, the user implements $g$ and $f$ and encapsulates those in a class that our framework is templated on. For instance, our Posit implementation for "exponent size" $m$ is listed in Appendix B. As can be seen, this class is also templated on the real arithmetic type [14] that serves as a proxy for the complete set of reals, $\mathbb{R}$. By default, this type is given by the long double $\mathrm{C}++$ type, which usually is implemented as 80 -bit extended precision. It may freely be substituted with higher precision types such as quad precision or MPFR [5], which allows for arbitrary precision.

\section{DISCUSSION AND CONCLUDING REMARKS}

We have presented a flexible framework for defining new number systems based on recursive bisection. While requiring the user to define only two simple functions, we show how many prior number systems can be expressed in our framework. Moreover, our framework provides a new perspective on how to define new number systems in an intuitive way via bracketing sequences-at a coarse scale-and local refinement-at a fine scale. We investigated properties of number systems derived through our framework and showed how to smoothly "interpolate" a given bracketing sequence using an associated cumulative distribution function that ultimately defines the coding scheme. This CDF provides for a natural refinement scheme that is compatible with the bracketing sequence.

Two drawbacks of our approach are the need to process one bit at a time and to perform computations-using an auxiliary arithmetic type-to generate bracketing and refinement points. This makes our framework unsuitable for applications that demand high performance. However, it allows for rapid prototyping and experimentation with new number systems without the implementation headache associated with complex bit manipulations that are error prone. Implementations that may require hundreds of lines of carefully crafted code are reduced to just two lines-regardless of the chosen precision. Once a good number system has been found for a target application, one may switch to a more optimized implementation that can be validated and certified to be correct using our framework. For low-precision types, one may, for instance, resort to lookup tables.

Due to space limitations, our study has no room for experimental evaluations of the various number systems. This is a fruitful topic for future work. We also point the interested reader to our earlier study [14], which evaluates in detail several of the representations presented here.

\section{ACKNOWLEDGMENTS}

This work was performed under the auspices of the U.S. Department of Energy by Lawrence Livermore National Laboratory under Contract DE-AC52-07NA27344 and was supported by the LLNL-LDRD Program under Project No. 17-SI-004.

\section{REFERENCES}

[1] 2008. IEEE Std 754-2008: IEEE Standard for Floating-Point Arithmetic

[2] Jon L. Bentley and Andrew C. Yao. 1976. An almost optimal algorithm for unbounded searching. Inform. Process. Lett. 5, 3 (1976), 82-87.

[3] George Bergman. 1957. A Number System with an Irrational Base. Mathematics Magazine 31, 2 (1957), 98-110.

[4] Peter Elias. 1975. Universal codeword sets and representations of the integers. IEEE Transactions on Information Theory 21, 2 (1975), 194-203.

[5] Laurent Fousse, Guillaume Hanrot, Vincent Lefèvre, Patrick Pélissier, and Paul Zimmermann. 2007. MPFR: A Multiple-precision Binary Floating-point Library with Correct Rounding. ACM Trans. Math. Software 33, 2 (2007), 13:1-13:15.

[6] Xavier Glorot and Yoshua Bengio. 2010. Understanding the difficulty of training deep feedforward neural networks. In Conference on Artificial Intelligence and Statistics. 249-256.

[7] John L. Gustafson. 2016. A Radical Approach to Computation with Real Numbers. Supercomputing Frontiers and Innovations 3, 2 (2016), 38-53.

[8] John L. Gustafson and Isaac T. Yonemoto. 2017. Beating Floating Point at its Own Game: Posit Arithmetic. Supercomputing Frontiers and Innovations 4, 2 (2017), 71-86.

[9] Hozumi Hamada. 1983. URR : Universal Representation of Real Numbers. New Generation Computing 1 (1983), 205-209.

[10] Nicholas J. Higham. 2002. Accuracy and Stability of Numerical Algorithms (second ed.). SIAM.

[11] Nick G. Kingsbury and Peter J. W. Rayner. 1971. Digital Filtering using Logarithmic Arithmetic. Electronic Letters 7, 2 (1971), 56-58.

[12] Donald E. Knuth. 1976. Mathematics and Computer Science: Coping with Finiteness. Science 194, 4271 (1976), 1235-1242.

[13] Siew Hoon Leong. 2017. NGA SoftPosit. https://gitlab.com/cerlane/SoftPosit.

[14] Peter Lindstrom, Scott Lloyd, and Jeffrey Hittinger. 2018. Universal Coding of the Reals: Alternatives to IEEE Floating Point. In Conference for Next Generation Arithmetic. 5:1-5:14.

[15] Robert Morris. 1971. Tapered Floating Point: A New Floating-Point Representation. IEEE Trans. Comput. C-20, 12 (1971), 1578-1579.

[16] David Salomon. 2007. Variable-Length Codes for Data Compression. SpringerVerlag.

[17] Isaac Yonemoto. 2017. FastSigmoid. https://github.com/Etaphase/FastSigmoids.jl. 


\section{A MATHEMATICA CODE}

(* decode a binary string, $x *$ )

decode[y_] := decode[StringReplace[y, $\{" 0 "->" 1 ", " 1 "->" 0 "\}, 1],\{$ Indeterminate, Indeterminate $\}]$

$\operatorname{decode}\left[y_{-},\left\{\min _{-}, \max _{-}\right\}\right]:=\operatorname{decode}[\operatorname{StringDrop}[y, 1], \operatorname{If}[\operatorname{StringTake}[y, 1]==" 0 ",\{\min , \operatorname{bisect}[\min , \max ]\},\{\operatorname{bisect}[\min , \max ], \max \}]]$

decode $\left[",,\left\{\min _{-}, \max _{-}\right\}\right]:=\min$

(* decode a p-bit signed integer, $y *$ )

decode $\left[y_{-}, p_{-}\right]:=\operatorname{decode}\left[\operatorname{IntegerString}\left[\operatorname{Mod}\left[y, 2^{\wedge} p\right], 2, p\right]\right]$

(* encode a real number, $x$, as a $p$-bit binary string *)

encode[Indeterminate, $\left.\mathrm{p}_{-}\right]:=\operatorname{IntegerString}\left[2^{\wedge}(\mathrm{p}-1), 2, \mathrm{p}\right]$

encode $\left[0, p_{-}\right]:=$IntegerString $[0,2, \mathrm{p}]$

encode $\left[x_{-}, p_{-}\right]:=$IntegerString $\left[B i t X o r\left[e n c o d e[x, p,\{\right.\right.$ Indeterminate, Indeterminate $\left.\left.\}, 0], 2^{\wedge}(p-1)\right], 2, p\right]$

encode $\left[x_{-}, p_{-},\left\{\min n_{-}, \max \right\}, y_{-}\right]:=\operatorname{If}[x<\operatorname{bisect}[\min , \max ], \operatorname{encode}[x, p-1,\{\min , \operatorname{bisect}[\min , \max ]\}, 2 y+0], \operatorname{encode}[x, p-1,\{b i \operatorname{sect}[\min , \max ], \max \}, 2 y+1]]$

encode $\left[x_{-}, \theta,\left\{\min _{-}, \max _{-}\right\}, y_{-}\right]:=\operatorname{round}[x,\{\min , \max \}, y]$

(* special rounding rules to avoid under- and overflow *)

round $\left[x_{-},\left\{\right.\right.$Indeterminate, $\left.\left.\max _{-}\right\}, y_{-}\right]:=y+1$

round $\left[x_{-},\left\{\right.\right.$min $_{-}$, Indeterminate $\left.\}, y_{-}\right]:=y+0$

round $\left[\mathrm{x}_{-},\left\{\min _{-}, 0\right\}, \mathrm{y}_{-}\right]:=\mathrm{y}+0$

$\operatorname{round}\left[x_{-},\left\{\theta, \max _{-}\right\}, y_{-}\right]:=y+1$

(* round $x$ to nearest value of $\{\min , \max \} *$ )

$\operatorname{round}\left[x_{-},\left\{\min _{-}, \max _{-}\right\}, y_{-}\right]:=\operatorname{If}[x<\operatorname{bisect}[\min , \max ], y+\theta$, If $[x>\operatorname{bisect}[\min , \max ], y+1, y+\operatorname{BitAnd}[y, 1]]]$

(* general bisection rules *)

bisect[Indeterminate, Indeterminate $]:=0$

bisect[0, Indeterminate] := 1

bisect[min_, max_?NonPositive] := -bisect[-max, $-\min ](*$ negation $*)$

bisect $\left[0, \max _{-}\right]:=\operatorname{bisect}\left[\max ^{\wedge}-1 \text {, Indeterminate }\right]^{\wedge}-1(*$ reciprocation $*)$

bisect[min, Indeterminate $]:=\mathrm{g}[\mathrm{min}](*$ bracketing $*)$

bisect $\left[\min _{-}, \max _{-}\right]:=f[\min , \max ](*$ refinement $*)$

(* power mean and hyper mean *)

pmean $\left[a_{-}, b_{-}, 0\right]:=\operatorname{Sqrt}[a b]$

pmean $\left[a_{-}, b_{-}, p_{-}\right]:=\left(\left(a^{\wedge} p+b^{\wedge} p\right) / 2\right)^{\wedge}(1 / p)$

hmean[a_?Negative, $b_{-} ?$ Negative $]:=-$ hmean $[-a,-b]$

hmean[a $\left.a_{-}, b_{-}\right]:=\operatorname{If}\left[a==0\|b==0\| 1 / 2<=a / b<=2,(a+b) / 2,2^{\wedge} h m e a n[\log 2[a], \log 2[b]]\right]$

Pick one representation and $m$-value below

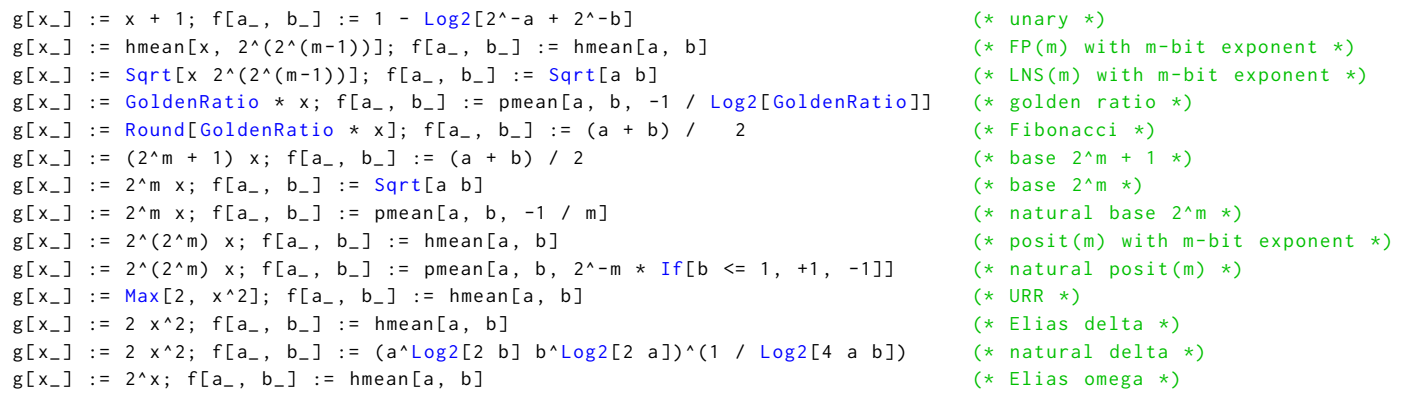

\section{B EXAMPLE $\mathrm{C}++$ CODE}

// Fibonacci representation

template <typename real = flex: :real>

struct Fibonacci \{

real operator ()$($ real $x)$ const $\{$ return std: : round $((1+\operatorname{std}:: \operatorname{sqrt}($ real $(5))) * x / 2)$; $\} / /$ generator $g(x)$ \};

// Elias delta representation

template <typename real = flex: :real>

struct EliasDelta \{

real operator ()$($ real $x)$ const $\{$ return real $(2) * x * x ;\}$ // generator $g(x)$ \}; 